\title{
Electric switching of magnetism in 2D
}

\author{
Combined electrostatic gating and magnetic fields switch a 2D atomic crystal from an antiferromagnetic to a \\ ferromagnetic state.
}

\section{Kenneth S. Burch}

lectronics based on magnetic materials have played a key role in sensors and data storage. Nonetheless, changing the magnetization direction typically requires generating magnetic fields, limiting the speed and size of devices. In nanoscale materials, various physical processes compete to determine the magnetic state: for example, magneto-elastic coupling, the interfacial breaking of symmetry, dipolar coupling, different exchange pathways, and so on ${ }^{1,2}$. Beyond the promise for new functionality and efficiency, nanoscale magnetism also opens the door to new states of matter. This was first suggested nearly five decades ago by theoretical results indicating the absence of long-range order and/or the presence of topological states in low dimensional magnets. Ultimately, these proposals rely on careful balancing between the various magnetic terms to allow continuous rotational symmetry (MerminWagner-Hohenberg theorem) or purely in-plane exchange (Kosterlitz-Thouless) ${ }^{1}$. As such, the complete destruction of magnetic order by reduction to two dimensions remains mostly elusive, but also promises to provide new superconductors, topologically nontrivial particles, or novel means for rapid switching of the magnetic state in devices.

Two-dimensional (2D) atomic crystals have recently emerged as an entirely new means to pursue these ideas ${ }^{3-6}$. Writing in Nature Nanotechnology, B. Huang et al. ${ }^{7}$ and S. Jiang et al. ${ }^{8}$ report the use of electrostatic gating to change not only the direction but the type of magnetic order in $\mathrm{CrI}_{3}$.

Shortly after first isolating graphene by mechanical exfoliation, the broad applicability of the technique to a range of materials was demonstrated. However, the use of this approach to magnetic materials has only just begun. Initial efforts succeeded in identifying the magnetic excitations $s^{5}$, presence of magnetic order via magneto-elastic coupling ${ }^{5,6}$, and Kerr rotation $^{3,4}$. As first pioneered in dilute magnetic semiconductors ${ }^{9}$, electrostatic gating has also succeeded in tuning the strength of the magnetic order ${ }^{10}$. Perhaps one of the most substantial surprises was
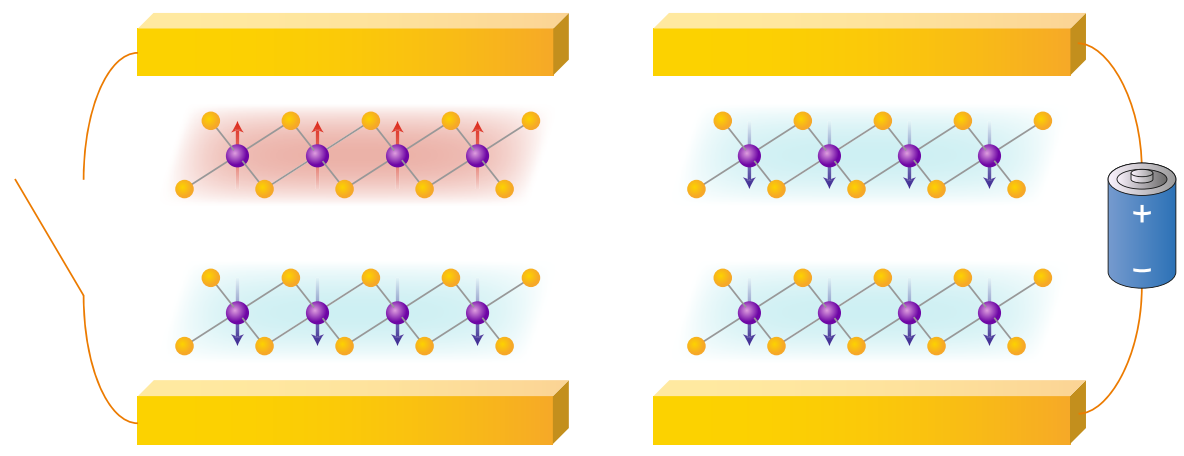

Fig. 1 | Schematic of electrically induced antiferromagnetic-to-ferromagnetic state switching in atomically thin $\mathrm{Crl}_{3}$.

the finding that the type of magnetic order (interlayer antiferromagnetism versus 3D ferromagnetism) is tuned via thickness ${ }^{3}$. This suggested a careful balancing of magnetic exchange terms, often seen in thin magnetic films, which has proven exceedingly difficult to tune controllably ${ }^{1,2}$.

Both sets of authors prepared bilayer $\mathrm{CrI}_{3}$ such that it could be gated (Fig. 1), as this previously showed an antiferromagnetic ground state with the two layers polarized opposite to one another. To monitor the magnetism, the change in reflectance between left and right circularly polarized light was employed (magneto circular dichroism, MCD), as well as the rotation of polarization on reflection (Kerr), and a technique widely used in thin magnetic films, AC-Kerr, to measure the susceptibility ${ }^{11}$. On applying a perpendicular magnetic field, $\mathrm{CrI}_{3}$ undergoes a metamagnetic transition to a fully polarized ferromagnetic state. Perhaps not surprising for a magnetic semiconductor ${ }^{2,9,10}$, the transition temperature could be modulated by inducing charge via electrostatic gating. More important for future technological and fundamental studies is the electrostatic modulation of the field required to cause a metamagnetic transition. Indeed, at a fixed magnetic field, the sample can be flipped from an antiferromagnetic state to a ferromagnetic one. This result strongly suggests that the electrostatic gating is tipping the balance between various exchange terms in the $2 \mathrm{D} \mathrm{CrI}_{3}$. These findings open the door to novel optoelectronic and magnetic devices where an electric field radically changes the material from having no net magnetization to a large and finite one. Furthermore, this provides a controllable means to tune the various exchange mechanisms in this material, moving us one step closer to realizing the dream of unlocking new phases by careful tuning of competing interactions in $2 \mathrm{D}$ magnets.

Kenneth S. Burch

Department of Physics, Boston College, Chestnut Hill, MA, USA.

e-mail:ks.burch@bc.edu

Published online: 23 May 2018

https://doi.org/10.1038/s41565-018-0165-4

References

1. De'Bell, K., MacIsaac, A. B. \& Whitehead, J. P. Rev. Mod. Phys. 72, 225-257 (2000)

2. Hellman, F. et al. Rev. Mod. Phys. 89, 25006 (2017).

3. Huang, B. et al. Nature 546, 270-273 (2017).

4. Gong, C. et al. Nature 546, 265-269 (2017).

5. Tian, Y. et al. 2D Mater. 3, 025035 (2016).

6. Lee, J.-U. et al. Nano Lett. 16, 7433-7438 (2016).

7. Huang, B. et al. Nat. Nanotech. https://doi.org/10.1038/s41565018-0121-3 (2018).

8. Jiang, S., Li, L., Wang, Z., Mak, K. F. \& Shan, J. Nat. Nanotech. https://doi.org/10.1038/s41565-018-0135-x (2018).

9. Ohno, H. et al. Nature 408, 944-946 (2000).

10. Deng, Y. et al. Preprint at https://arxiv.org/abs/1803.02038 (2018).

11. Arnold, C. S., Dunlavy, M. \& Venus, D. Rev. Sci. Instrum. 68 4212-4216 (1997). 